\title{
Venovenous extracorporeal membrane oxygenation in patients with COVID-19 respiratory failure
}

J. W. Awori Hayanga, MD, MPH, ${ }^{\mathrm{a}}$ Subhasis Chatterjee, MD, ${ }^{\mathrm{b}}$ Bo Soo Kim, MD, HelenMari Merritt-Genore, DO, ${ }^{\mathrm{d}}$ Rita Carrie Karianna Milewski, MD, PhD, ${ }^{\mathrm{e}}$ Jonathan W. Haft, MD, ${ }^{\mathrm{f}}$ and Rakesh C. Arora, MD, $\mathrm{PhD}^{\mathrm{g}}$

The high level of human-to-human transmissibility of severe acute respiratory syndrome coronavirus 2 (SARSCoV-2) and the severity of coronavirus disease 2019 (COVID-19) have perpetuated an international public health emergency. Indeed, this pandemic has evolved into the greatest health challenge of the 21 st century. Virus entry is mediated by the structural spike membrane protein, which plays a significant role in host cell receptor recognition and membrane fusion. Angiotensin-converting enzyme 2 serves as the functional culprit host cell receptor.

The COVID-19 pandemic has tested the resilience of the global health infrastructure and unleashed a slew of novel strategies and technological advances in the armamentarium against this viral threat. Like the experience with influenza pandemic of the early 2000s, extracorporeal support has served as a rescue option favored in the face of failing conventional therapy. The clinical experience with extracorporeal membrane oxygenation (ECMO) thus far has been framed through the lens of single-center, nonrandomized reports that vary in both methodology and outcomes. ${ }^{2-5}$ Severity of disease may be estimated using the World Health Organization classification system, which assigns a grade from 1 to 9 in order of increasing severity. ${ }^{6}$ There is not yet a consensus ECMO-specific scoring system, and to this end, the Murray, PRESERVE, $^{4}$ and RESP $^{5}$ scores each remain applicable in the quantification of severity in this cohort, although not necessarily specific to COVID-19 or its prognosis.

From the ${ }^{\text {a Department }}$ of Cardiothoracic and Vascular Surgery, West Virginia University Medicine, Morgantown, WVa; ${ }^{\mathrm{b}}$ Divisions of General and Cardiothoracic Surgery, Department of Surgery, Baylor College of Medicine, Houston, Tex; ${ }^{\mathrm{c} D i v i s i o n}$ of Pulmonary and Critical Care Medicine, Johns Hopkins Medicine, Baltimore, Md; ${ }^{\mathrm{d}}$ Heart Consultants, Methodist Physicians Clinic, Omaha, Neb; ${ }^{\mathrm{e}}$ Department of Cardiac Surgery, Yale School of Medicine, New Haven, Conn; ${ }^{\mathrm{f}}$ Department of Cardiac Surgery, Michigan Medicine, University of Michigan, Ann Arbor, Mich; and ${ }^{\mathrm{g}}$ Section of Cardiac Surgery, Department of Surgery, Max Rady College of Medicine, University of Manitoba, Winnipeg, Manitoba, Canada.

Received for publication Aug 1, 2021; revisions received Sept 15, 2021; accepted for publication Sept 28, 2021; available ahead of print Oct 12, 2021.

Address for reprints: J. W. Awori Hayanga, MD, MPH, Department of Cardiovascular and Thoracic Surgery, West Virginia University, 1 Medical Center Dr, Morgantown, WV 26506 (E-mail: jeremiah.hayanga@wvumedicine.org).

J Thorac Cardiovasc Surg 2023;165:212-7

$0022-5223 / \$ 36.00$

Copyright (c) 2021 by The American Association for Thoracic Surgery

https://doi.org/10.1016/j.jtcvs.2021.09.059

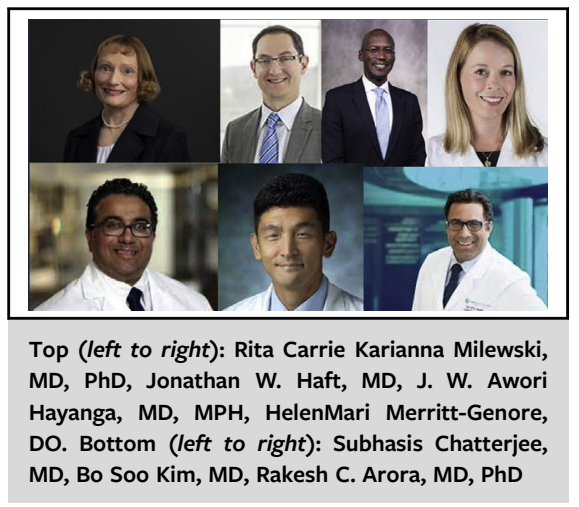

CENTRAL MESSAGE

Adjustments to candidacy and

traditional approaches to extra-

corporeal support may mitigate

mortality during a pandemic.

See Commentaries on pages 218 and 219.

In the absence of broad consensus, management strategies have been both dynamic and variable. ${ }^{7,8}$ Here we proffer a distillation of expert opinion in the management of extracorporeal support in COVID-19 patients, drawing on our collective high-volume multi-institutional experience.

\section{Assessing Candidacy}

The high mortality from the COVID-19 virus often prompted deviation from previously held norms regarding a candidacy that had been established in previous landmark trials, such as the EOLIA $^{9}$ and CESAR $^{10}$ trials. Based on our collective experience, we have observed a shift in 3 domains. First, candidacy now tends to more greatly favor younger patients, prioritizing those age $<50$ years with only single organ dysfunction. Second, there is greater willingness to shorten the interval from intubation to cannulation. Success is more likely in those cannulated after $<3$ days of mechanical ventilation while meeting the EOLIA criteria (partial pressure of $\mathrm{O}_{2}$ /fraction of inspired $\mathrm{O}_{2}[\mathrm{PF}]$ ratio $<50 \mathrm{~mm} \mathrm{Hg}$ for $>3$ hours, or $<80 \mathrm{~mm} \mathrm{Hg}$ for $>6$ hours, or $\mathrm{pH}<7.25$ with partial pressure of $\mathrm{CO}_{2}>60 \mathrm{~mm} \mathrm{Hg}$ for $>6$ hours). ${ }^{9}$ Finally, an array of novel adjunctive pharmacologic therapies, including antiviral and monoclonal antibodies and the use 
TABLE 1. Precannulation guide to estimating survival in COVID -19

\begin{tabular}{lccr}
\hline \multicolumn{1}{c}{ Factor } & High probability of survival & Medium probability of survival & Low probability of survival \\
\hline Age, $\mathrm{y}$ & $<45$ (1 point) & 45 to 55 (2 points) & $>55(3$ points $)$ \\
Days of mechanical ventilation & $<3$ (1 point) & 3 to 76 (2 points $)$ & $>7(3$ points $)$ \\
Systemic comorbidities & 0 (1 point) & 1 to 2 (2 points $)$ & $>2(3$ points $)$ \\
\hline
\end{tabular}

Total score: high survival, 1 to 3 points; medium survival, 4 to 6 points; low survival, 6 to 9 points.

of extracorporeal cytoreductive techniques, have been introduced as adjuncts to ECMO support. The sickest patients typically present with a median APACHE II score ${ }^{2}$ of score of $>20$, a Murray score of $^{3} \geq 3$, and/or a SOFA score of $^{7}$ $\geq 8$. In our collective experience, patients sick enough to require venoarterial ECMO from the outset typically developed early multisystem failure and were almost uniformly unsalvageable. ${ }^{2}$ We proffer a matrix to provide a rudimentary estimate of survival (Table 1) and a list of factors associated with adverse outcomes (Table 2).

\section{Equipment and Hardware}

ECMO technology has evolved rapidly over the past decade and involves an assembly of components that are separate or integrated into a single platform. Centrifugal pumps have supplanted previously used roller pumps. ${ }^{11-13}$ The exposure of blood to air exacerbates inflammatory and coagulation activation and is associated with the end-organ injury observed during prolonged runs of cardiopulmonary bypass. ${ }^{14}$ This activation is exacerbated by the COVIDinduced cytokine storm regardless of the type of pump used, and indeed is likely to be partially responsible for the increased incidence of circuit clotting that often characterizes extracorporeal support in these patients irrespective of the type or level of anticoagulation provided.

The distribution and supply chain of ECMO equipment faltered under the weight of escalating demand during the pandemic and manufacturing could not keep pace. In the United States, where Food and Drug Administration approval for ECMO circuits had hitherto been restricted to 6-hour use, the pandemic provided the impetus for an acceleration in the regulatory process for components under evaluation. ${ }^{15,16}$ The CentriMag (Levitronix, Waltham, Mass), Rotaflow (Getinge, Göteborg, Sweden), CardioHelp (Maquet Cardiopulmonary, Hirrlingen, Germany), and Revolution (LivaNova, London, UK) devices, which had previously dominated the market, have made way for the NovaLung pump (NovaLung, Hechingen, Germany) introduced in mid-2020. This system, along with the Nautilus oxygenator (Medtronic, Minneapolis, Minn) and Breethe OXY-1 system (Abiomed, Danvers, Mass), have benefited from comparatively expedited federal clearance. ${ }^{1,9}$ Many centers struggled to acquire the prerequisite number of circuits and components at the height of the surge and often were unsuccessful, no doubt to the detriment of a multitude of patients.

\section{Cannulation Considerations}

The vast majority of patients who require ECMO treatment for severe COVID-19 are supported on venovenous ECMO. ${ }^{4}$ Indeed, in keeping with the dismal results that characterized the early experience with venoarterial ECMO, it is reasonable to restrict support to only those who require venovenous support and consider vasopressor-dependent cardiogenic shock an absolute

TABLE 2. Factors associated with adverse outcomes, information deficits, and investigative priorities in venovenous extracorporeal membrane oxygenation

Factors associated with adverse outcome

Male sex

Age $>50$ years

Vasopressor-dependent cardiogenic shock

More than 2 systemic comorbidities

(eg, renal failure, liver failure)

Right ventricular failure

Support $>50$ days

Unvaccinated status

\section{Information deficits}

Does obesity portend a poor prognosis?

Can cytoreductive therapies improve survival?

Should the interval between mechanical ventilation and cannulation be limited to 72 hours?

Should we prone patients with COVID-19 while on ECMO?

Should we consider the need for venoarterial ECMO an absolute contraindication?

Is right ventricular dysfunction reversible in COVID-19 ARDS?

Should we limit the duration of support to 50 days?

\section{Investigative priorities}

Use of cytoreductive techniques in treating acute severe illnesses

Can these techniques be used to treat septic shock, postcardiotomy shock, and other viral illnesses?

Pharmacokinetics of antiviral therapies on ECMO

How much drug is lost to the circuit, and what dosing would be most optimal?

Correlation of cytokine storm with shedding of replication competent virus

Do high circulating inflammatory markers mean the same thing during and after the period of infectivity? 
contraindication. For venovenous ECMO support, dual-site peripheral cannulation involving the femoral vein $(25 \mathrm{~F}-27 \mathrm{~F}$ multistage) and the internal jugular vein (20F-22F) has been a common strategy of choice. Cannulation may be performed expeditiously at the bedside in this manner, which obviates the need for patient transportation to the operating room, allows rapid initiation, and minimizes staff exposure. Furthermore, securing the cannula and tubing to the skin with interrupted silk suture also permits ambulation.

Alternatively, a large-bore $28 \mathrm{~F}$ to $32 \mathrm{~F}$ double-lumen bicaval cannula (Avalon Elite; Getinge or Crescent; Medtronic) may be inserted into the right internal jugular vein. However, these cannulas require fluoroscopy and transesophageal echocardiography guidance for safe placement in the inferior vena cava. ${ }^{17,18}$ The peripheral cannulation strategy performed in a negative-pressure intensive care unit (ICU) room was pertinent early in the pandemic when no treatment or vaccine had yet been identified and personal protective equipment (PPE) was in short supply. The ProtekDuo (LivaNova), an alternative dual-lumen cannula designed with a drainage port in the right atrium and an infusion port in the main pulmonary artery, is positioned across the tricuspid and pulmonary valves and is an alternative cannulation strategy. This cannula provides the additional benefit of right ventricular support, which may benefit selected patients, but it has not yet received universal endorsement. ${ }^{18,19}$

\section{Mechanical Ventilation Considerations}

It is postulated that the SARS-CoV-2 virus exists as 2 distinct phenotypic entities ( $\mathrm{H}$ and $\mathrm{L}$ types) with other multiple variants. ${ }^{20}$ As such, COVID-19 acute respiratory distress syndrome (ARDS) was initially deemed to always require high levels of positive end-expiratory pressure (PEEP) for lung recruitment and oxygenation. This is now thought to be true only for patients with the H phenotype, whose lungs have high elastance (low compliance), high lung weight by computed tomography scan, and high response to PEEP. In contrast, patients with the L phenotype (low elastance, low lung weight, low response to PEEP) require an alternative ventilatory strategy with higher tidal volumes and lower levels of PEEP. ${ }^{21}$ The L type likely reflects a distinct vascular pathology secondary to diffuse alveolar damage. Alternatively, the 2 subtypes may represent a continuum of the same disease process, with possible unidirectional progression from the $\mathrm{L}$ phenotype to the $\mathrm{H}$ phenotype. $^{20}$ An original assumption that COVID-19 ARDS was identical to other sources of ARDS has now been disputed. The use of high PEEP settings increases the risk of barotrauma, with clinical and radiologic evidence of pneumomediastinum and pneumothoraces. As such, once on ECMO, a deliberate reduction in the level of PEEP is often necessary. ${ }^{22}$ Nonetheless, ventilator strategies have been a heterogeneous mix influenced by prevailing experience and equipment.
The use of proning in patients with a $\mathrm{PF}$ ratio $<150$ has been considered standard of care but also may be applicable for nonintubated patients on high-flow nasal cannula who are able to self-prone and can do so for several hours on a daily basis. ${ }^{23,24}$ For the sickest patients with a PF ratio $<100$, short-term use of neuromuscular blockade with the addition of inhaled nitric oxide (iNO) may be used as a matter of routine. ${ }^{25}$ When the PF ratio does not improve within the first 8 to 16 hours, or indeed if it worsens, it may be reasonable to proceed with cannulation to avoid a longer than 4-day lapse from intubation to best minimize the duration of barotrauma.

Once a patient is on ECMO, complete lung rest is the goal and can typically be accomplished using a fraction of inspired $\mathrm{O}_{2}$ of $30 \%$, PEEP support of 10 , and respiratory rate of 10 . However, these settings are not necessarily possible in all patients owing to the risk of complete derecruitment, which itself may be deleterious. Instead, recruitment maneuvers can be used in combination with alternative ventilator modes, such as airway pressure release ventilation, tailoring the approach to the individual patient, further guided by esophageal manometry if necessary. Ventilation is further facilitated by an aggressive approach to early tracheostomy, which may be routinely performed percutaneously in the ICU as early as ECMO day 4, as extubation might not necessarily be possible. Early tracheostomy facilitates weaning and cessation of sedation and paralytics and accelerates the time to mobilization, decreasing the burden of critical illness polyneuropathy and tissue injury incurred from prolonged recumbency. It is typically coupled with nasoenteric or percutaneous gastrostomy feeding tube placement. Liberal use of pulmonary vasodilation is common, and iNO is favored over inhaled epoprostenol owing to its association with fewer ventilator circuit interruptions and lower exposure risk to staff. Admittedly, neither neuromuscular blockade nor iNO has demonstrated a clear survival benefit in recent trials, but each has some utility in optimizing the sickest ventilated patients. ${ }^{26,27}$

\section{Specific Considerations for the Obese Patient}

Managing the super morbidly obese ECMO patient (body mass index $>45$ ) on a ventilator is challenging. Poor compliance of the chest wall decreases the accuracy of the measurements of lung mechanics, rendering difficult any precise determination of safe PEEP level. ${ }^{28}$ Esophageal balloon manometry may be used to estimate transmural pressures across alveolar walls, to minimize harmful distending pressures and prevent lung decruitment. Esophageal manometry can help determine optimal PEEP, which in turn can stent alveoli open, preventing atelectrauma. Once an optimal PEEP is identified, pressure control may be adjusted to maintain peak airway pressure $<30 \mathrm{~cm} \mathrm{H}_{2} \mathrm{O}$. If PEEP determined in this fashion is high enough to precipitate a peak pressure $>30 \mathrm{~cm} \mathrm{H}_{2} \mathrm{O}$ in the setting of driving pressure $<10 \mathrm{~cm} \mathrm{H}_{2} \mathrm{O}$ (plateau pressure minus PEEP) and tidal 
volume between 2 and $4 \mathrm{cc} / \mathrm{kg}$ of ideal body weight, then the optimal PEEP is titrated down further to achieve a peak airway pressure of $<30 \mathrm{~cm} \mathrm{H}_{2} \mathrm{O}$. As lung function improves, PEEP should continue to be measured and titrated down as tolerated. Once PEEP has been weaned to $10 \mathrm{~cm} \mathrm{H}_{2} \mathrm{O}$ and lungs remain recruited, the ventilator settings can then be weaned with a goal of minimizing driving pressure and liberating the patient from positive pressure. A body mass index $>50$ also poses a threat to achieving adequate flow on ECMO and may potentially compromise mesenteric and peripheral circulation, and thus some centers have adopted this cutoff as a relative contraindication to conventional mechanical ventilator support.

\section{Pharmacologic Adjuncts}

The pharmacologic management strategies for COVID-19 have incorporated novel approaches, including repurposed antivirals, cytokine inhibitors, immunomodulators, anticoagulants, and new antiviral vaccines. These strategies have varied over time and with prevailing evidence from multiple large, randomized trials. To this end, various agents have risen and fallen in profile, including hydroxychloroquine, tocilizumab, ivermectin, remdesivir, convalescent plasma, and various monoclonal antibodies. ${ }^{29-34}$ Almost all have been subjected to enthusiastic endorsement as well as tempered skepticism. Notably, the impact of 4 repurposed antivirals has been challenged by the recent World Health Organization Solidarity trial. ${ }^{35}$ Nonetheless, steroids, particularly dexamethasone in the RECOVERY trial, have withstood scrutiny and have been associated with improved survival. ${ }^{36-40}$

The use of cytoreductive techniques using either CytoSorb (CytoSorbents, Monmouth Township, NJ) or plasma exchange has drawn great interest, albeit with varying degrees of acceptance. These strategies extract inflammatory mediators from the blood up to $60 \mathrm{kDa}$ in size using bead pore capture. ${ }^{41}$ CytoSorb can be used only within the confines of emergency use authorization from the Food and Drug Administration. Therapy is initiated at the time of cannulation, incorporating the cartridge in the ECMO circuit like the management of continuous renal replacement therapy and continued for the first 72 hours. One of the authors endorses the liberal use of plasma exchange in patients with D-dimer $>3000$ and ferritin $>1000$ (using $2 \mathrm{~L}$ replaced by a 50-50 mix of plasma and 5\% albumin or fresh frozen plasma). With this, a $30 \%$ reduction in target cytokines can be achieved within 72 hours. The use of IL- 6 level as a target for titration may be delayed because the sample typically must be sent out for external laboratory estimation.

Although heparin remains the anticoagulant of choice in most ECMO programs, bivalirudin is increasingly gaining prominence. Anticoagulation protocols have a similar target maintenance partial thromboplastin time of 50 to 70; however, most centers still use heparin for bolus administration at the time of cannulation, and it is also preferentially used with CytoSorb. Regardless of choice, anticoagulation may be safely interrupted to accommodate other adjunctive procedures, such as percutaneous tracheostomy and percutaneous endoscopic gastrostomy placement, both of which also may be performed at the bedside.

\section{Capacity and Staffing}

The COVID-19 pandemic has created unique health care worker staffing shortages and resource challenges. Although the geographic distribution of the pandemic was asymmetric, no large community has been spared. Elective surgical cases had to be postponed to preserve ventilators, ICU beds, and PPE. Many ICUs triaged personnel to newly created COVID-19 units while simultaneously merging other, non-COVID ICUs. ${ }^{42}$ COVID-19 put front-line workers at risk of infection, not just from patients, but also from coworkers. As the pandemic progressed, unprecedented nursing shortages forced many ICUs to reallocate personnel, reduce capacity, or spend magnitudes higher on salaries for temporary nursing staff. Deficits were further exacerbated by health care personnel becoming infected or exposed, necessitating periods of quarantine; school and childcare facility closures that forced working parents to stay home; new opportunities for temporary employment with significantly higher pay; and significant emotional burnout from an unprecedented and relentless challenge. ${ }^{43}$

A threshold of volume and utilization has been identified beyond which a nursing-based model becomes more cost conscious than relying on perfusionists at the bedside. ${ }^{44}$ COVID-19 has shown that a pandemic can exert such a strain on staffing ratios that creative solutions, incentivized remuneration models, hazard pay, and work hour flexibility likely will be necessary considerations for any future high-volume ECMO efforts. The CESAR trial endorsed the transfer of patients to ECMO centers, and many advocate clustering patients within expert ECMO centers for improved outcomes. Others, however, have endorsed grouping ECMO patients within institutions themselves to alleviate staffing shortages and bed capacity within facilities.

Because ECMO centers typically constitute the "hub" in the hub-and-spoke model, patients are often transferred in from lower-capacity external critical care settings to the ECMO centers. In these models of care delivery, the early identification and use of transfer protocols allow for safe and expeditious triaging of patients who may benefit from ECMO to the nearest facility with the best capacity to offer support. ECMO transport teams may initiate support at referring institutions and use transport and safety checklists to facilitate the process.

\section{Resource Management}

The highest mortality was observed in patients with advanced age or immunosuppression. Most patients who died were ultimately removed from ECMO for futility. The definition of futility varied from weeks to months, 
depending on prevailing opinion. Many centers developed contingency plans, incorporated ethics committee members into decision making, and even chose to ration scarce resources such as ventilators, dialysis circuits, and ECMO circuits. Some centers modified standard ACLS algorithms restricting cardiopulmonary resuscitation in patients with COVID-19, given the near- $100 \%$ mortality rate. ${ }^{21}$ As the pandemic unfolded, resources became limited-first ECMO circuits, then ECMO specialists and nurses. It became necessary to select patients most likely to benefit from support. As overall ECMO capacity diminished, contraindications to ECMO tended to become more stringent, consistent with Extracorporeal Life Support Organization guidelines. ${ }^{8}$ This is a dynamic process, and individual centers are encouraged to define tiers of contingency and crisis capacity to adjudicate need in the context of forecasted reductions in capability and resources. To preserve PPE, for example, isolation precautions typically can be lifted on all patients at 20 days after infection, beyond which the likelihood of the presence of replication-competent virus is negligible. These decisions may be guided by high cycle thresholds, as an index of low volume of replicating viral genetic material and, by extension, of low infectivity.

The coordination of care and management between health systems offering ECMO support (both adult and pediatric) in a single region can be of great utility amid a disaster. We suggest a triage protocol as shown in Figure 1. Collaborative coalitions of this nature were attempted and proved of great utility in the states of Texas and Pennsylvania. In one author's personal experience, a designated "ECMO regional coordinator" was assigned to manage equipment and inventory, closely monitor fluxes in available staffing and beds, and steward the dissemination of critical information between the directors of ECMO programs within the given region. In Texas, this also facilitated evaluation for transplantation.

\section{CONCLUSIONS}

The COVID-19 pandemic has been the greatest health challenge of the 21st century. The current estimate of survival is estimated at just over $50 \%{ }^{7}$ In view of the relative novelty of the SARS-Cov-2 virus, there is a paucity of evidence-based recommendations on which to base management or to guide patient management —indeed there are several knowledge gaps that require scientific inquiry for further elaboration (Table 2 ), together with a list of plausible areas of inquiry (Table 2).

The pandemic has tested the resilience of the global health infrastructure but also has unleashed opportunities for novel approaches in mitigating viral threats that indeed may become a template for the future. By making iterative adjustments to traditional considerations in extracorporeal support pertaining to candidacy, capacity, and resource allocation, ECMO programs can maximize the ability to respond to pandemics now and in the future. Centers within the same region should

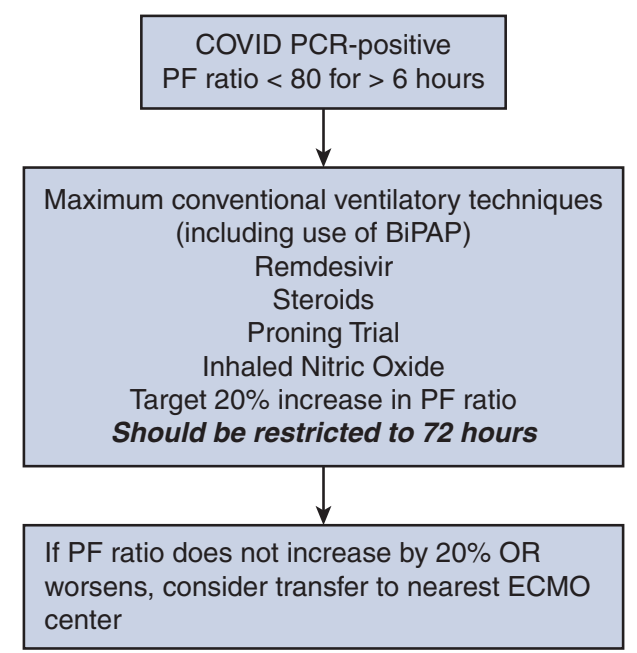

FIGURE 1. Triage protocol for resource scarce centers without ECMO. COVID PCR, Severe acute respiratory syndrome coronavirus 2 polymerase chain reaction test; $P F$ ratio, partial pressure of $\mathrm{O}_{2} /$ fraction of inspired $\mathrm{O}_{2}$ ratio; $B I P A P$, bilevel positive airway pressure; $O R$, operating room; $E C M O$, extracorporeal membrane oxygenation.

advisedly identify common inclusion and exclusion criteria and titrate outreach and scope of care based on resources and capacity. There should be a low threshold for including ethics teams to guide equitable distribution of care and navigate end-of life decisions. During a pandemic and in the absence of defined consensus or evidence-based recommendations, candidacy is predicated not on the probability of choosing the right individual, but instead on the consequences of choosing the wrong one. We should choose the patient who is so sick that cannulation is necessary but not so sick that decannulation is not possible.

\section{Conflict of Interest Statement}

Dr Arora has received honoraria from AVIR Pharma Inc, Edwards Lifesciences and Abbott Nutrition. Dr MerrittGenore serves as a consultant for AtriCure. All other authors reported no conflicts of interest.

The Journal policy requires editors and reviewers to disclose conflicts of interest and to decline handling or reviewing manuscripts for which they may have a conflict of interest. The editors and reviewers of this article have no conflicts of interest.

\section{References}

1. Zamorano Cuervo N, Grandvaux N. ACE2: evidence of role as entry receptor for SARS-CoV-2 and implications in comorbidities. ELife. 2020;9:e61390.

2. Raasveld SJ, Delnoij TSR, Broman LM, Lansink-Hartgring AO, Hermans G, De Troy E, et al. Extracorporeal membrane oxygenation in patients with COVID-19: an international multicenter cohort study. J Intensive Care Med. 2021;36:910-7.

3. Schmidt M, Hajage D, Lebreton G, Monsel A, Voiriot G, Levy D, et al. Extracorporeal membrane oxygenation for severe acute respiratory distress syndrome associated with COVID-19: a retrospective cohort study. Lancet Respir Med. 2020;8:1121-31.

4. Jacobs JP, Stammers AH, Louis JS, Awori Hayanga JW, Firstenberg MS, Mongero LB, et al. Multi-institutional analysis of 100 consecutive patients with COVID-19 and severe pulmonary compromise treated with extracorporeal membrane oxygenation: outcomes and trends over time. ASAIO J. 2021;67:496-502. 
5. Nguyen NT, Sullivan B, Sagebin F, Hohmann SF, Amin A, Nahmias J. Analysis of COVID-19 patients with acute respiratory distress syndrome managed with extracorporeal membrane oxygenation at US academic centers. Ann Surg. 2021;274:40-4.

6. World Health Organization. Clinical management of severe acute respiratory infection (SARI) when COVID-19 disease is suspected: interim guidance, 13 March 2020. Available at: https://apps.who.int/iris/handle/10665/331446. Accessed October 25, 2021.

7. Barbaro RP, MacLaren G, Boonstra PS, Iwashyna TJ, Slutsky AS, Fan E, et al. Extracorporeal membrane oxygenation support in COVID-19: an international cohort study of the Extracorporeal Life Support Organization Registry. Lancet. 2020;396:1071-8.

8. Badulak J, Antonini MV, Stead CM, Shekerdemian L, Raman L, Paden ML, et al. Extracorporeal membrane oxygenation for COVID-19: updated 2021 guidelines from the Extracorporeal Life Support Organization. ASAIO J. 2021;67:485-95.

9. Combes A, Hajage D, Capellier G, Demoule A, Lavoué S, Guervilly C, et al. Extracorporeal membrane oxygenation for severe acute respiratory distress syndrome. N Engl J Med. 2018;378:1965-75.

10. Peek GJ, Mugford M, Tiruvoipati R, Wilson A, Allen E, Thalanany MM, et al. Efficacy and economic assessment of conventional ventilatory support versus extracorporeal membrane oxygenation for severe adult respiratory failure (CESAR): a multicentre randomised controlled trial. Lancet. 2009;374:1351-63.

11. Palanzo D, Qiu F, Baer L, Clark JB, Myers JL, Ündar A. Evolution of the extracorporeal life support circuitry. Artif Organs. 2010;34:869-73.

12. Halaweish I, Cole A, Cooley E, Lynch WR, Haft JW. Roller and centrifugal pumps: a retrospective comparison of bleeding complications in extracorporeal membrane oxygenation. ASAIO J. 2015;61:496-501.

13. Bottrell S, Bennett M, Augustin S, Thuys C, Schultz B, Horton A, et al. A comparison study of haemolysis production in three contemporary centrifugal pumps. Perfusion. 2014;29:411-6.

14. Millar JE, Fanning JP, McDonald CI, McAuley DF, Fraser JF. The inflammatory response to extracorporeal membrane oxygenation (ECMO): a review of the pathophysiology. Crit Care. 2016;20:387.

15. Modern Healthcare. U.S. medical supply chains failed, and COVID deaths followed; October 6, 2020. Available at: https://www.modernhealthcare.com/ supply-chain/us-medical-supply-chains-failed-and-covid-deaths-followed. Accessed July 14, 2021

16. Beninger P. COVID-19: regulatory landscape of medicinal and medical device products for human use. Clin Ther. 2020;42:1444-50.

17. Kuhl T, Michels G, Pfister R, Wendt S, Langebartels G, Wahlers T. Comparison of the Avalon dual-lumen cannula with conventional cannulation technique for venovenous extracorporeal membrane oxygenation. Thorac Cardiovasc Surg. 2015;63:653-62.

18. Badu B, Cain MT, Durham LA III, Joyce LD, Sundararajan S, Gaglianello N, et al. A dual-lumen percutaneous cannula for managing refractory right ventricular failure. ASAIO J. 2020;66:915-21.

19. Ravichandran AK, Baran DA, Stelling K, Cowger JA, Salerno CT. Outcomes with the Tandem Protek Duo dual-lumen percutaneous right ventricular assist device. ASAIO J. 2018;64:570-2.

20. Gattinoni L, Chiumello D, Caironi P, Busana M, Romitti F, Brazzi L, et al. COVID-19 pneumonia: different respiratory treatments for different phenotypes? Intensive Care Med. 2020;46:1099-102.

21. Marini JJ, Gattinoni L. Management of COVID-19 respiratory distress. JAMA. 2020;323:2329-30.

22. Walkey AJ, Del Sorbo L, Hodgson CL, Adhikari NKJ, Wunsch H, Meade MO, et al. Higher PEEP versus lower PEEP strategies for patients with acute respiratory distress syndrome: a systematic review and meta-analysis. Ann Am Thorac Soc. 2017;14(Suppl_4):S297-303.

23. Slessarev M, Cheng J, Ondrejicka M, Arntfield R. Critical Care Western Research Group. Patient self-proning with high-flow nasal cannula improves oxygenation in COVID-19 pneumonia. Can J Anaesth. 2020;67:1288-90.

24. Guérin C, Reignier J, Richard JC, Beuret P, Gacouin A, Boulain T, et al. Prone positioning in severe acute respiratory distress syndrome. N Engl J Med. 2013; 368:2159-68.

25. Lotz C, Muellenbach RM, Meybohm P, Mutlak H, Lepper PM, Rolfes CB, et al. Effects of inhaled nitric oxide in COVID-19-induced ARDS-is it worthwhile? Acta Anaesthesiol Scand. 2021;65:629-32.
26. National Heart, Lung, and Blood Institute PETAL Clinical Trials Network, Moss M, Huang DT, Brower RG, Ferguson ND, Ginde AA, et al. Early neuromuscular blockade in the acute respiratory distress syndrome. $N$ Engl J Med 2019;380:1997-2008.

27. Taylor RW, Zimmerman JL, Dellinger RP, Straube RC, Criner GJ, Davis K Jr, et al. Low-dose inhaled nitric oxide in patients with acute lung injury: a randomized controlled trial. JAMA. 2004;291:1603-9.

28. Magder S, Slobod D, Assanangkornchai N. Mechanical ventilation in the obese patient: compliance, pleural pressure, and driving pressure. Am J Respir Crit Care Med. 2021;203:534-6.

29. Ibáñez S, Martínez O, Valenzuela F, Silva F, Valenzuela O. Hydroxychloroquine and chloroquine in COVID-19: should they be used as standard therapy? Clin Rheumatol. 2020;39:2461-5.

30. Rosas IO, Bräu N, Waters M, Go RC, Hunter BD, Bhagani S, et al. Tocilizumab in hospitalized patients with severe Covid-19 pneumonia. N Engl J Med. 2021 384:1503-16.

31. Bryant A, Lawrie TA, Dowswell T, Fordham EJ, Mitchell S, Hill SR, et al. Ivermectin for prevention and treatment of COVID-19 infection: a systematic review, meta-analysis, and trial sequential analysis to inform clinical guidelines. Am J Ther. 2021;28:e434-60.

32. Beigel JH, Tomashek KM, Dodd LE, Mehta AK, Zingman BS, Kalil AC, et al. Remdesivir for the treatment of Covid-19-final report. N Engl J Med. 2020 383:1813-26.

33. Rajendran K, Krishnasamy N, Rangarajan J, Rathinam J, Natarajan M Ramachandran A. Convalescent plasma transfusion for the treatment of COVID-19: systematic review. J Med Virol. 2020;92:1475-83.

34. Marovich M, Mascola JR, Cohen MS. Monoclonal antibodies for prevention and treatment of COVID-19. JAMA. 2020;324:131-2.

35. WHO Solidarity Trial Consortium. Repurposed antiviral drugs for COVID-19-interim WHO Solidarity Trial results. $N$ Engl J Med. 2021;384 497-511.

36. RECOVERY Collaborative Group, Horby P, Lim WS, Emberson JR, Mafham M, Bell JL, et al. Dexamethasone in hospitalized patients with Covid-19. N Engl J Med. 2021;384:693-704.

37. WHO Rapid Evidence Appraisal for COVID-19 Therapies (REACT) Working Group, Sterne JAC, Murthy S, Diaz JV, Slutsky AS, Villar J, et al. Association between administration of systemic corticosteroids and mortality among critically ill patients with COVID-19: a meta-analysis. JAMA. 2020;324:1330-41.

38. Fadel R, Morrison AR, Vahia A, Smith ZR, Chaudhry Z, Bhargava P, et al. Early short-course corticosteroids in hospitalized patients with COVID-19. Clin Infect Dis. 2020;71:2114-20.

39. Edalatifard M, Akhtari M, Salehi M, Naderi Z, Jamshidi A, Mostafaei S, et al Intravenous methylprednisolone pulse as a treatment for hospitalised severe COVID-19 patients: results from a randomised controlled clinical trial. Eur Respir J. 2020;56:2002808.

40. Wang Y, Jiang W, He Q, Wang C, Liu B, Zhou P, et al. Early, low-dose and shortterm application of corticosteroid treatment in patients with severe COVID-19 pneumonia: single-center experience from Wuhan, China. medRxiv. March 12, 2020; https://doi.org/10.1101/2020.03.06.20032342 [preprint].

41. Supady A, Weber E, Rieder M, Lother A, Niklaus T, Zahn T, et al. Cytokine adsorption in patients with severe COVID-19 pneumonia requiring extracorporeal membrane oxygenation (CYCOV): a single-centre, open-label, randomised, controlled trial. Lancet Respir Med. 2021;9:755-62.

42. Chatterjee S, Anton JM, Rosengart TK, Coselli JS. Cardiac surgery during the COVID-19 sine wave: preparation once, preparation twice. A view from Houston. J Card Surg. 2021;36:1615-23.

43. Spetz J. There are not nearly enough nurses to handle the surge of coronavirus patients: here's how to close the gap quickly. Health Affairs Blog; March 21, 2020. Available at: https://www.healthaffairs.org/do/10.1377/hblog20200327. 714037/full/. Accessed June 19, 2021.

44. Dhamija A, Kakuturu J, Schauble D, Hayanga HK, Jacobs JP, Badhwar V, et al. Outcome and cost of nurse-led versus perfusionist-led extracorporeal membrane oxygenation. Ann Thorac Surg. May 24, 2021 [Epub ahead of print].

Key Words: extracorporeal membrane oxygenation, COVID-19, adult respiratory distress syndrome 